\title{
THE EFFECTS OF THE PEAK-PEAK CORRELATION ON THE PEAK MODEL OF HIERARCHICAL CLUSTERING
}

\author{
Alberto Manrique ${ }^{1}$, Andreu Raig ${ }^{1}$, José María Solanes ${ }^{1}$, Guillermo González-Casado², Paul \\ Stein $^{1}$, and Eduard Salvador-Solé ${ }^{1}$ \\ E-mail: alberto@faess2.am.ub.es, araig@mizar.am.ub.es, solanes@pcess1.am.ub.es, \\ guille@coma.upc.es, stein@pcess2.am.ub.es, eduard@faess0.am.ub.es
}

\begin{abstract}
In two previous papers a semi-analytical model was presented for the hierarchical clustering of halos via gravitational instability from peaks in a random Gaussian field of density fluctuations. This model is better founded than the extended Press-Schechter model, which is known to agree with numerical simulations and to make similar predictions. The specific merger rate, however, shows a significant departure at intermediate captured masses. The origin of this was suspected as being the rather crude approximation used for the density of nested peaks. Here, we seek to verify this suspicion by implementing a more accurate expression for the latter quantity which accounts for the correlation among peaks. We confirm that the inclusion of the peak-peak correlation improves the specific merger rate, while the good behavior of the remaining quantities is preserved.
\end{abstract}

Subject headings: cosmology: theory - galaxies: clustering - galaxies: formation

\section{INTRODUCTION}

Today it is widely accepted that the formation of cosmic relaxed objects proceeds via gravitational instability from small to large scales. This clustering process can be studied in detail, following accurately the dynamics of collapse by means of N-body simulations, and statistically, by approximate semi-analytical models which enable a swifter and more efficient exploration of the parameter space.

The most complete semi-analytical models developed to date are those by Lacey \& Cole (1993, 1994, hereafter LC93 and LC94), and by Manrique \& Salvador-Solé (1995, 1996, Papers I and II).

\footnotetext{
${ }^{1}$ Departament d'Astronomia i Meteorologia, Universitat de Barcelona, Avda. Diagonal 647, 08028 Barcelona, Spain,

${ }^{2}$ Departament de Matemàtica Aplicada II, Universitat Politècnica de Catalunya, Pau Gargallo 5, 08028 Barcelona, Spain.
} 
All the quantities in both models are derived from the statistics of the filtered random Gaussian density field, $\delta(\mathbf{x}) \equiv[\rho(\mathbf{x})-\langle\rho\rangle] /\langle\rho\rangle$, of cold matter encountered at an arbitrary initial epoch, $t_{i}$, after recombination when fluctuations are still linear. Similarly, in each model the dynamics of dissipationless collapse is approximated by the simple spherical model. These two models differ however in the following aspects.

Lacey \& Cole's (LC) model is based on the Press \& Schechter (1974, PS) heuristic prescription for the mass functions of halos, duly extended to obtain the conditional mass function of halos at a given epoch subject to having a larger mass at a later time (Bower 1991; Bond et al. 1991). $N$-body simulations show that this model gives highly satisfactory predictions (LC94). In spite of this remarkable success, there remains the caveat that the seeds of halos are arbitrary points and their associated collapsing clouds undetermined (possibly disconnected) regions around them (cf. LC93). Besides, the formation and destruction of halos are arbitrarily and ambiguously defined, leading to poorly motivated formation and destruction times (see Paper II).

The model developed by Manrique \& Salvador-Solé uses the new CUSP (ConflUent System of Peak trajectories) formalism, which enables one to follow the filtering evolution of peaks in the density contrast vs. filtering scale ( $\delta$ vs. $R$ ) diagram at $t_{i}$, assumed to trace the mass evolution of halos. This model is better founded than the LC model insofar as high peaks are good seeds of massive halos (Bond \& Myers 1996) and do follow spherical collapse (Bernardeau 1994). On the other hand, it provides a natural distinction between accretion and merger which makes the formation and destruction of dark halos well-defined and uniquely determined.

As shown in Papers I and II both models provide very similar results for the mass function, the mass increase rate, and the merger rate at large captured masses. However, notable departures were observed in this latter rate at intermediate captured masses. This discrepancy was suspected as being caused by the rather crude approximation used in the CUSP model for the density of nested peaks, in connection with the cloud-in-cloud correction. In the present paper we seek to verify this point. We improve the nesting correction by taking into account the correlation among peaks and investigate the effects this has on the CUSP model. In $\S 2$ we review the foundations of the CUSP model developed in Papers I and II. In $\S 3$ we derive a more accurate approximation for

the density of nested peaks and implement it in $\S 4$. Our results are summarized in $\S 5$. Comoving lengths are assumed throughout the paper.

\section{THE CUSP MODEL}

Based on the spherical collapse model, the peak ansatz states that there is a correspondence between non-nested peaks of fixed linear overdensity $\delta_{c}$ in the density field smoothed on scale $R$ at any arbitrary initial epoch $t_{i}$ and quasi-steady halos of mass $M$ at a time $t \geq t_{i}$. The mass $M$ of the spherical collapsing cloud, or equivalently, of the final halo associated with a peak is an increasing function of the filtering scale $R$, whereas $\delta_{c}$ is a decreasing function of $t$. The mass function of halos 
at $t, N(M, t) d M$, is therefore simply given by the density of peaks with $\delta_{c}$ on scales $R$ to $R+d R$, $N_{p k}\left(R, \delta_{c}\right) d R$, properly corrected for nesting and transformed to the variables $M$ and $t$ through the relations $R(M)$ and $\delta_{c}(t)$.

The approximate expression for the nesting correction used in Paper I was (see next section for justification)

$$
N(R, \delta)=N_{p k}(R, \delta)-\int_{R}^{\infty} \frac{M\left(R^{\prime}\right)}{\langle\rho\rangle} N\left(R^{\prime}, \delta\right) N_{p k}\left(R, \delta \mid R^{\prime}, \delta\right) d R^{\prime} .
$$

In equation (1),$\langle\rho\rangle$ is the mean density of the universe, $M\left(R^{\prime}\right) /\langle\rho\rangle$ is the volume, to 0th order in $\delta$, of the collapsing cloud associated with a peak with $\delta$ on scale $R^{\prime}$, and $N_{p k}\left(R, \delta \mid R^{\prime}, \delta\right) d R$ is the conditional density of peaks with $\delta$ on scales $R$ to $R+d R$ subject to being located at a point with $\delta$ on scale $R^{\prime}>R$. This equation is a Volterra-type integral equation of the second kind for the scale function $N(R, \delta)$ of non-nested peaks, which can be readily solved in the standard way from the known functions $N_{p k}(R, \delta)$ and $N_{p k}\left(R, \delta \mid R^{\prime}, \delta\right)$ derived in Paper I (eqs. [11] and [17]).

The functions $R(M)$ and $\delta_{c}(t)$ required to obtain $N(M, t)$ from $N(R, \delta)$ are fixed by simple consistency arguments (see Paper I)

$$
R(M)=\frac{1}{q}\left(\frac{3 M}{4 \pi\langle\rho\rangle}\right)^{1 / 3}, \quad \delta_{c}(t)=\delta_{c e}(t) \frac{D\left(t_{i}\right)}{D(t)} .
$$

In the above equation $D(t)$ is the linear growth factor at the considered collapse time $t$ and $\delta_{c e}(t)$ is the density contrast for collapse at $t$ linearly extrapolated to $t$, both functions being dependent on the particular cosmogony assumed. Constants $q$ and $\delta_{c 0} \equiv \delta_{c e}\left(t_{0}\right)$ are therefore the only free parameters of the model. For each pair of values of these parameters, the filtering process translates into a different mass evolution of halos, with only one couple of values (approximately) recovering the evolution encountered in real gravitational clustering. The correct values of these two parameters can be obtained by adjusting the mass function predicted by the model to the mass function resulting from $N$-body simulations at some given time, for example at $t_{0}$ (this automatically leads to a similarly good fit at any other time; see $\S 4$ ).

The remaining statistical quantities predicted by the model rely on the definitions of accretion and merger, and halo formation and destruction. Since in hierarchical clustering halos grow constantly by capturing other halos, the fact that they are quasi-steady systems implicitly presumes that some of the captures are tiny enough for the steady state of the capturing systems not to be essentially altered. Notable captures yielding a transient departure from steadiness establish then the frontier between the initial and final quasi-steady systems. Accretion is therefore defined as any apparently continuous and derivable mass increase along the temporal series of quasi-steady systems subtending at each step the mass of those which precede them. In contrast, a merger is any appreciable discontinuity in the mass increase along such a temporal series causing a significant departure from the steadiness of the growing system at that step. A halo is said to survive as long as it evolves by accretion, whereas when it merges it is said to be destroyed. Note that when a halo 
is captured by one that is more massive it merges. However, the capturing halo may just accrete it if the relative captured mass is small enough. Only those events in which all the initial halos merge and are destroyed give rise to the formation of new halos. As shown in Paper II, the assumed correspondence between halos and non-nested peaks allows one to naturally identify the filtering processes at $t_{i}$ tracing all the preceding processes, as well as to derive their respective rates and characteristic times (see $\S 4$ ).

\section{A MORE ACCURATE EXPRESSION FOR NESTING}

In the original version of the CUSP model the density distribution around peaks was approximated by top-hat spheres. In addition, the conditional density of peaks with $\delta$ on scales $R$ to $R+d R$, having $\delta^{\prime}$ on $R^{\prime}>R$ and being located within a peak on that larger scale, was approximated by the conditional density of peaks with $\delta$ on scales $R$ to $R+d R$ simply having $\delta^{\prime}$ on scale $R^{\prime}$. Under these circumstances, the density of peaks with $\delta$ on scales $R$ to $R+d R$ located within non-nested peaks with $\delta^{\prime}$ on scales $R^{\prime}$ to $R^{\prime}+d R^{\prime}\left(R^{\prime}>R\right)$ takes the simple form

$$
N_{p k}^{n e s t}\left(R \rightarrow R^{\prime}, \delta \rightarrow \delta^{\prime}\right) d R d R^{\prime}=\frac{M\left(R^{\prime}\right)}{\langle\rho\rangle} N\left(R^{\prime}, \delta^{\prime}\right) d R^{\prime} N_{p k}\left(R, \delta \mid R^{\prime}, \delta^{\prime}\right) d R .
$$

Equation (3) leads, for $\delta^{\prime}=\delta$, to the scale function of non-nested peaks (eq. [1]) and, for $\delta^{\prime}=\delta-d \delta$, to the density of peaks which become nested in an infinitesimal decrement of $\delta$ (eq. 9 below), respectively tracing the mass function of halos and the density of halos merging into more massive ones. Any inaccuracy in this expression, therefore, translates into these two quantities and any other that is related. To improve equation (3), we would need to drop the top-hat approximation and take into account the peak-peak correlation. Approximate expressions for this latter quantity have been obtained by Bardeen et al. (1986; hereafter BBKS) and Regös \& Szalay (1995). Unfortunately, these are not useful for our purposes; being only valid for large separations compared to the filtering scale, while what is needed here is the peak-peak correlation for separations up to $R^{\prime}$.

An alternative, more accurate, expression for the density of nested peaks can be obtained, however, from the conditional probability function $P_{\tilde{\delta}}(\delta, x, R, r)$ of finding the density contrast $\tilde{\delta}$ on scale $R$ at a separation $r$ from a peak with $\delta$ and curvature $x$ on that scale (averaged over the orientations of the second order derivative tensor) provided by BBKS. Using this conditional probability function we can write

$$
\begin{array}{r}
N_{p k}^{n e s t}\left(R \rightarrow R^{\prime}, \delta \rightarrow \delta^{\prime}\right) d R d R^{\prime}=\frac{M\left(R^{\prime}\right)}{\langle\rho\rangle} \\
\quad N\left(R^{\prime}, \delta^{\prime}\right) d R^{\prime} \int_{0}^{1} d r 3 r^{2} \int_{0}^{\infty} d x^{\prime} P_{x^{\prime}}\left(\delta^{\prime}, R^{\prime}\right) \\
\times \int_{-\infty}^{\infty} d \tilde{\delta} P_{\tilde{\delta}}\left(\delta^{\prime}, x^{\prime}, R^{\prime}, r\right) N_{p k}\left(R, \delta \mid R^{\prime}, \tilde{\delta}\right) d R
\end{array}
$$

with $r$ in units of $q R$, and $P_{x^{\prime}}\left(\delta^{\prime}, R^{\prime}\right) \equiv N\left(R^{\prime}, x^{\prime}, \delta^{\prime}\right) / N\left(R^{\prime}, \delta^{\prime}\right)$ the curvature probability function for non-nested peaks with $\delta^{\prime}$ on scales $R^{\prime}$ to $R^{\prime}+d R^{\prime}$. Expression (4) takes into account the real density distribution around peaks and, since this includes the distribution of neighboring peaks 
(on every scale), it also accounts for the peak-peak correlation as desired. It is not exact however, because the conditional mean density of peaks with $\delta$ on scales $R$ to $R+d R$ subject to having $\tilde{\delta}$ on scale $R^{\prime}$ at a distance $r$ from a peak with $\delta^{\prime}$ and $x^{\prime}$ on $R^{\prime}$ is approximated by $N_{p k}\left(R, \delta \mid R^{\prime}, \tilde{\delta}\right) d R$, the conditional mean density of peaks with $\delta$ on scales $R$ to $R+d R$ subject to just having $\tilde{\delta}$ on scale $R^{\prime}$.

In the Appendix we detail the calculations that allow us to write equation (4) in a compact form identical to equation (3), but for the new function $N_{p k}^{n e s t}\left(R, \delta \mid R^{\prime}, \delta^{\prime}\right)$ (eq. A13]) instead of the conditional density function $N_{p k}\left(R, \delta \mid R^{\prime}, \delta^{\prime}\right)$. In Figure 1 we plot these two estimates of the density of nested peaks. As can be seen, they are very different for $R^{\prime}$ close to $R$ while they asymptotically coincide for $R^{\prime} \gg R$. This is well understood. There is no correlation among peaks on very different scales. Thus the conditional mean density of peaks with $\delta$ on scales $R$ to $R+d R$ subject to being located at a point with $\tilde{\delta}$ on scale $R^{\prime}$ at a distance $r$ from a peak with $\delta^{\prime}$ and $x^{\prime}$ on the same scale is very well approximated, in this case, by the simple conditional mean density $N_{p k}\left(R, \delta \mid R^{\prime}, \tilde{\delta}\right) d R$ used in equation (đ) leading to $N_{p k}^{n e s t}\left(R, \delta \mid R^{\prime}, \delta^{\prime}\right)$. On the other hand, the spherical top-hat approximation is also very good in the limit for $R^{\prime} \gg R$. Hence, not only is the new function $N_{p k}^{n e s t}\left(R, \delta \mid R^{\prime}, \delta^{\prime}\right)$ entirely accurate at this limit, but it also coincides with the former function $N_{p k}\left(R, \delta \mid R^{\prime}, \delta^{\prime}\right)$.

\section{EFFECTS ON THE CUSP MODEL}

All the quantities predicted by the new version of the CUSP model are readily obtained, following the same derivations as in Papers I and II, by simply replacing the function $N_{p k}\left(R, \delta \mid R^{\prime}, \delta^{\prime}\right)$ by $N_{p k}^{n e s t}\left(R, \delta \mid R^{\prime}, \delta^{\prime}\right)$. This leads to the following expressions.

The mass function of halos is

$$
N(M, t)=N\left(R, \delta_{c}\right) \frac{d R}{d M}
$$

with $N(R, \delta)$ the scale function of non-nested peaks at $\delta$, solution of the Volterra equation

$$
N(R, \delta)=N_{p k}(R, \delta)-\int_{R}^{\infty} \frac{M\left(R^{\prime}\right)}{\langle\rho\rangle} N\left(R^{\prime}, \delta\right) N_{p k}^{n e s t}\left(R, \delta \mid R^{\prime}, \delta\right) d R^{\prime}
$$

In Figure 2 we compare this mass function with that obtained in the LC model. The parameters $\delta_{c 0}$ and $q$ entering in the CUSP model were fixed by adjusting it to the PS mass function in the range $5 \times 10^{13} \mathrm{M}_{\odot} \leq M \leq 10^{15} \mathrm{M}_{\odot}$ at $z=0$, where the latter gives a very good fit to the results of $N$-body simulations. Both functions are very similar for massive halos at any redshift. Only for small masses does the CUSP mass function predict slightly fewer halos than that of the PS. Note also that the large differences between the new and old approximations for nesting at $R^{\prime} \sim R$ (Fig. 1) have no appreciable effect on the mass function: the original and new solutions being almost exactly superimposed. This indicates that the mass function is particularly insensitive, in the mass range of Figure 2, to the peak-peak correlation. 
The instantaneous mass accretion rate for halos of mass $M$ at $t$ is

$$
r_{\text {mass }}^{a}(M, t)=\overline{\frac{\left(x^{-1}\right)}{\sigma_{2} R}} \frac{d M}{d R}\left|\frac{d \delta_{c}}{d t}\right|,
$$

with $\sigma_{2}$ the second order spectral moment (eq. A6 $)$, and $\overline{\left(x^{-1}\right)}$ the average inverse curvature for the distribution function $P_{x}(\delta, R)$. There is no similar prediction in the LC model which does not differentiate accretion from merger. Only the total mass increase rates arising from the two models can be compared (see below). In Figure 3 we plot the accretion rates obtained from the original and new versions of the CUSP model. Once again, both solutions are almost fully superimposed showing that this rate is also insensitive to the peak-peak correlation.

The instantaneous specific merger rate for halos of mass $M$ at $t$ per infinitesimal range of the resulting masses $M^{\prime}>M$ is

$$
r^{m}\left(M \rightarrow M^{\prime}, t\right)=\frac{\mathbf{N}^{m}\left(R \rightarrow R^{\prime}, \delta_{c}\right)}{N\left(R, \delta_{c}\right)} \frac{d R^{\prime}}{d M^{\prime}}\left|\frac{d \delta_{c}}{d t}\right|,
$$

where $\mathbf{N}^{m}\left(R \rightarrow R^{\prime}, \delta_{c}\right) d R d R^{\prime} d \delta$ is the density of peaks with $\delta_{c}$ on scales $R$ to $R+d R$ becoming nested into non-nested peaks with $\delta_{f}=\delta_{c}-d \delta$ on scales $R^{\prime}$ to $R^{\prime}+d R^{\prime}$

$$
\mathbf{N}^{m}\left(R \rightarrow R^{\prime}, \delta\right)=-\left.\frac{M\left(R^{\prime}\right)}{\langle\rho\rangle} \partial_{\delta_{f}}\left[N\left(R^{\prime}, \delta_{f}\right) N_{p k}^{n e s t}\left(R, \delta \mid R^{\prime}, \delta_{f}\right)\right]\right|_{\delta_{f}=\delta} .
$$

To compare this specific merger rate with that yielded by the LC model we must take into account the different merger definitions adopted in the two models. In the LC model, merger is any mass capture experienced by halos. In contrast, in the CUSP model only notable captures are considered true mergers, while small captures contribute to accretion. As shown in Figure 4, the CUSP specific merger rate (using both the original and new approximations for the density of nested peaks) fully recovers that of the $\mathrm{LC}$ in the asymptotic regime at large $\Delta M / M \equiv\left(M^{\prime}-M\right) / M$, but notably deviates for small $\Delta M / M$. This departure is in the expected direction: for small mass captures the specific merger rate increases monotonically in the LC model, while it shows an abrupt cutoff in the CUSP model.

From Figure 4 we also see that the original and new versions of the CUSP model now give markedly different solutions. They coincide at very large $\Delta M / M$, owing to the similarity of the density of nested peaks at $R^{\prime} \gg R$, but the old solution begins to decline, deviating from the LC solution, at $\Delta M / M$ much greater than unity. This implies that some smooth distinction between tiny and notable captures is already operating at $\Delta M / M \gg 1$, which is meaningless. This is not the case for the new solution which keeps closer to that of the LC model until $\Delta M / M \sim 1$, then rapidly falls off implying the existence of a sharp effective frontier between tiny and notable captures at $\Delta M / M \lesssim 1$. Nonetheless, the new solution is not fully satisfactory: it becomes slightly negative for $\Delta M / M$ smaller than the effective threshold for merger, and the latter tends to be unreasonably small or large for extreme values of $M$. This shows the shortcomings of the new approximation used for the density of nested peaks. 
All the remaining quantities predicted by the CUSP model are determined by the three preceding ones which consequently fix the behavior of the whole model. The instantaneous total mass increase rate for halos of mass $M$ at $t, r_{\text {mass }}(M, t)$, is equal to the mass accretion rate (eq. [7]) plus the mass merger rate, defined as

$$
r_{\text {mass }}^{m}(M, t)=\int_{M}^{\infty} \Delta M r^{m}\left(M \rightarrow M^{\prime}, t\right) d M^{\prime} .
$$

The instantaneous destruction rate (or global merger rate) of halos of mass $M$ at $t$ is

$$
r^{d}(M, t)=\int_{M}^{\infty} r^{m}\left(M \rightarrow M^{\prime}, t\right) d M^{\prime},
$$

while their instantaneous formation rate take the form

$$
r^{f}(M, t)=\frac{\mathbf{N}^{f}\left(R, \delta_{c}\right)}{N\left(R, \delta_{c}\right)}\left|\frac{d \delta_{c}}{d t}\right|
$$

where $\mathbf{N}^{f}(R, \delta) d R d \delta$ is the density of non-nested peaks appearing from $\delta$ to $\delta-d \delta$,

$$
\begin{aligned}
\mathbf{N}^{f}(R, \delta)= & -\partial_{\delta} N_{p k}(R, \delta)+\left.\int_{R}^{\infty} \frac{M\left(R^{\prime}\right)}{\langle\rho\rangle} N\left(R^{\prime}, \delta\right) \partial_{\delta^{\prime}} N_{p k}^{n e s t}\left(R, \delta^{\prime} \mid R^{\prime}, \delta\right)\right|_{\delta^{\prime}=\delta} d R^{\prime} \\
& +\partial_{R} \mathcal{N}_{p k}(\delta, R)-\int_{R}^{\infty} \frac{M\left(R^{\prime}\right)}{\langle\rho\rangle} N\left(R^{\prime}, \delta\right) \partial_{R} \mathcal{N}_{p k}^{n e s t}\left(\delta, R \mid \delta, R^{\prime}\right) d R^{\prime} \\
& +\frac{M(R)}{\langle\rho\rangle} N(R, \delta) \lim _{R^{\prime} \rightarrow R} \mathcal{N}_{p k}^{n e s t}\left(\delta, R \mid \delta, R^{\prime}\right)
\end{aligned}
$$

(note the error in the expression quoted in Paper II), with $\mathcal{N}_{p k}(\delta, R)$ and $\mathcal{N}_{p k}^{n e s t}\left(\delta, R \mid \delta, R^{\prime}\right)$ defined for a fixed $R$ instead of a fixed $\delta$ (see Paper I). To see that expressions (12) and (13) can be written in terms of the first three quantities above, one must simply substitute $N(M, t), r_{\text {mass }}^{a}(M, t)$, and $r^{d}(M, t)$ (eqs. [5], [7], and [11]) into the conservation equation

$$
\frac{d \ln N}{d t}=r^{f}[M(t), t]-r^{d}[M(t), t]-\left.\partial_{M} r_{m a s s}^{a}(M, t)\right|_{M=M(t)},
$$

for the number density of halos per unit mass along mean accretion tracks, $M(t)$, solutions of the differential equation

$$
\frac{d M}{d t}=r_{\text {mass }}^{a}[M(t), t]
$$

Finally, the distributions (and typical values) of formation and destruction times can be readily inferred from the expression giving the cumulative spatial number density of halos at $t_{i}$ with masses from $M\left(t_{i}\right)$ to $M\left(t_{i}\right)+\delta M\left(t_{i}\right)$ surviving until $t_{f}$

$$
N_{\text {sur }}\left(t_{f}\right)=N\left[M\left(t_{i}\right), t_{i}\right] \delta M\left(t_{i}\right) \exp \left\{-\int_{t_{i}}^{t_{f}} r^{d}\left[M\left(t^{\prime}\right), t^{\prime}\right] d t^{\prime}\right\}
$$

with $M(t)$ the mass at $t$ of such halos calculated along their mean accretion tracks, and

$$
\delta M\left(t_{i}\right)=\delta M\left(t_{f}\right) \exp \left[-\left.\int_{t_{i}}^{t_{f}} \partial_{M} r_{\text {mass }}^{a}\left(M, t^{\prime}\right)\right|_{M=M\left(t^{\prime}\right)} d t^{\prime}\right]
$$


the element of mass at $t_{i}$ evolving, through mean accretion tracks, into $\delta M\left(t_{f}\right)$ at $t_{f}$. Indeed, by taking $t_{i}=t_{0}$ and $t_{f}=t$ in equation (16) we obtain the distribution of destruction times of halos at $t_{0}$ with masses between $M_{0}$ and $M_{0}+\delta M_{0}$, with $\delta M_{0}$ arbitrarily small,

$$
\Phi_{d}(t) \equiv-\frac{1}{N_{\text {sur }}\left(t_{0}\right)} \frac{d N_{\text {sur }}}{d t}=r^{d}[M(t), t] \exp \left\{-\int_{t_{0}}^{t} r^{d}\left[M\left(t^{\prime}\right), t^{\prime}\right] d t^{\prime}\right\}
$$

while, by taking into account that the cumulative spatial number density of those halos at $t_{0}$ which preexist at $t<t_{0}, N_{\text {pre }}(t)$, coincides with the cumulative spatial number density of halos at $t_{i}=t$ surviving until $t_{f}=t_{0}$, equations (14) and (17) lead to the distribution of formation times

$$
\Phi_{f}(t) \equiv \frac{1}{N_{\text {pre }}\left(t_{0}\right)} \frac{d N_{\text {pre }}}{d t}=r^{f}[M(t), t] \exp \left\{-\int_{t}^{t_{0}} r^{f}\left[M\left(t^{\prime}\right), t^{\prime}\right] d t^{\prime}\right\} .
$$

\section{CONCLUSIONS}

We have investigated the effects that the inclusion of the spatial correlation among peaks has on the CUSP model. The predictions for the mass function of halos, their mass accretion rate, and their specific merger rate for large captured masses, are very similar to those arising from the original version of this model, which were already satisfactory. On the contrary, the specific merger rate at intermediate masses changes appreciably, now becoming more consistent with the corresponding prediction by the LC model. We, therefore, confirm that the poor behavior shown by this latter quantity in the original version of the CUSP model was not intrinsic to its foundations, but due to the rather crude approximation originally used for the density of nested peaks. A more accurate expression for the peak-peak correlation than that used here is, however, required for the predicted specific merger rate to be fully satisfactory.

An interesting characteristic of the CUSP model is that it distinguishes between accretion and merger, which allows one to unambiguously define the formation and destruction of halos respectively as the last and next merger they experience. Mergers are those mass captures restructuring the systems, and hence, establishing their morphological properties until a new merger takes place. Such a distinction is therefore especially well suited for investigating the origin of the morphology of halos and, by extension, that of galaxies and galaxy clusters. Since the PS formalism involves much simpler expressions than those appearing in the peak theory, implementing that distinction in the former would have the added advantage of simplicity. The results of the present work suggest how it should be done. Such a modification of the LC model is used, in Salvador-Solé, Solanes, \& Manrique (1997), to study the origin of the correlation between halo concentration and halo mass recently found in cosmological $N$-body simulations (Navarro, Frenk, \& White 1996).

The present work has been supported by the Dirección General de Investigación Científica y Técnica under contract PB96-0173. P.S. has benefited from a grant of the Swiss Science Foundation. 


\section{APPENDIX: DENSITY OF NESTED PEAKS}

The same reasoning leading to equation (四) allows us to write its restriction to peaks with curvature $x$ to $x+d x$ on scale $R, \delta$ on scales $R$ to $R+d R$, and $\delta^{\prime}$ on scales $R^{\prime}$ to $R^{\prime}+d R^{\prime}$

$$
\begin{aligned}
N_{p k}^{n e s t}\left(x, R \rightarrow R^{\prime}, \delta \rightarrow \delta^{\prime}\right) d x d R d R^{\prime} & =\frac{M\left(R^{\prime}\right)}{\langle\rho\rangle} N\left(R^{\prime}, \delta^{\prime}\right) d R^{\prime} \int_{0}^{1} d r 3 r^{2} \int_{0}^{\infty} d x^{\prime} P_{x^{\prime}}\left(\delta^{\prime}, R^{\prime}\right) \\
& \times \int_{-\infty}^{\infty} d \tilde{\delta} P_{\tilde{\delta}}\left(\delta^{\prime}, x^{\prime}, R^{\prime}, r\right) N_{p k}\left(x, R, \delta \mid R^{\prime}, \tilde{\delta}\right) d x d R
\end{aligned}
$$

In the preceding expression we have taken the volume of the collapsing clouds associated with peaks delimited by a top-hat window with radius proportional to the filtering scale $R$.

Likewise, the density function of non-nested peaks with $\delta$ on scales $R$ to $R+d R$ and curvature between $x$ and $x+d x$ satisfies the relation

$$
N(x, R, \delta)=N_{p k}(x, R, \delta)-\int_{R}^{\infty} N_{p k}^{n e s t}\left(x, R \rightarrow R^{\prime}, \delta \rightarrow \delta\right) d R^{\prime},
$$

similar to equation (6). From equations (41), (A1), and (A2) we have

$$
\begin{aligned}
P_{x}(\delta, R)=\frac{N_{p k}(x, R, \delta)}{N(R, \delta)}\left[1-\int_{R}^{\infty} d R^{\prime}\right. & \frac{M\left(R^{\prime}\right)}{\langle\rho\rangle} N\left(R^{\prime}, \delta\right) \int_{0}^{1} d r 3 r^{2} \int_{0}^{\infty} d x^{\prime} P_{x^{\prime}}\left(\delta, R^{\prime}\right) \\
& \left.\times \int_{-\infty}^{\infty} d \tilde{\delta} P_{\tilde{\delta}}\left(\delta, x^{\prime}, R^{\prime}, r\right) \frac{N_{p k}\left(x, R, \delta \mid R^{\prime}, \tilde{\delta}\right)}{N_{p k}(x, R, \delta)}\right] .
\end{aligned}
$$

Under the approximation $P_{x}(\delta, R)=N_{p k}(x, R, \delta) / N_{p k}(R, \delta)$, the curvature moments for this probability function are accurate to within a few percent. Since the function $N_{p k}(x, R, \delta) / N_{p k}(R, \delta)$ is bell-shaped, relatively symmetrical, and close to a Gaussian it can be further approximated by a normal distribution with identical mean and variance, $\bar{x}$ and $(\Delta x)^{2}$. In taking this approximation we will also extend the domain of integration over $x^{\prime}$ in equation (A1) to the whole range $-\infty<x^{\prime}<\infty$. This does not introduce any appreciable error since $\bar{x}$ is considerably greater than $3 \Delta x$ for any reasonable values of $\delta$ and $R$ and any realistic spectrum.

The distribution function $P_{\tilde{\delta}}(\delta, x, R, r)$ is a normal distribution with mean and variance equal to $(\mathrm{BBKS})$

$$
\overline{\delta_{x}(r)}=\frac{\gamma \delta}{1-\gamma^{2}}\left(\frac{\psi}{\gamma}+\frac{\nabla^{2} \psi}{u^{2}}\right)-\frac{x \sigma_{0}}{1-\gamma^{2}}\left(\gamma \psi+\frac{\nabla^{2} \psi}{u^{2}}\right)
$$

and

$$
\left[\Delta \delta_{x}(r)\right]^{2}=\sigma_{0}^{2}\left\{1-\frac{1}{\left(1-\gamma^{2}\right)}\left[\psi^{2}+\left(2 \gamma \psi+\frac{\nabla^{2} \psi}{u^{2}}\right) \frac{\nabla^{2} \psi}{u^{2}}\right]-5\left(\frac{3 \psi^{\prime}}{u^{2} r}-\frac{\nabla^{2} \psi}{u^{2}}\right)^{2}-\frac{3\left(\psi^{\prime}\right)^{2}}{\gamma u^{2}}\right\} .
$$

In the above expressions, $\psi \equiv \xi(r) / \xi(0)$, with $r$ in units of $q R$ and $\xi(r)$ the mass correlation function on scale $R, \psi^{\prime} \equiv d \psi / d r, u^{2} \equiv(q R)^{2} \sigma_{2} / \sigma_{0}, \gamma \equiv \sigma_{1}^{2} /\left(\sigma_{0} \sigma_{2}\right)$, and

$$
\sigma_{i}^{2}(R) \equiv \int_{0}^{\infty} \frac{k^{2(i+1)}}{2 \pi^{2}} P(k) \exp \left(-k^{2} R^{2}\right) d k
$$


with $P(k)$ the power spectrum of the density fluctuations.

Given the normal character of both $P_{x^{\prime}}$ and $P_{\tilde{\delta}}$, the integration over $x^{\prime}$ on the right hand-side of equation (4) can be performed analytically, the result being

$$
\begin{aligned}
& N_{p k}^{n e s t}\left(R \rightarrow R^{\prime}, \delta \rightarrow \delta^{\prime}\right) d R d R^{\prime}=\frac{M\left(R^{\prime}\right)}{\langle\rho\rangle} N\left(R^{\prime}, \delta^{\prime}\right) d R^{\prime} \\
& \quad \times \int_{0}^{1} d r 3 r^{2} \int_{-\infty}^{\infty} d \tilde{\delta} P_{\tilde{\delta}}\left(\delta^{\prime}, R^{\prime}, r\right) N_{p k}\left(R, \delta \mid R^{\prime}, \tilde{\delta}\right) d R .
\end{aligned}
$$

The new function $P_{\tilde{\delta}}(\delta, R, r)$, equal to the probability function of finding the value $\tilde{\delta}$ at a separation $r$ from a peak with $\delta$ in the density field smoothed on scale $R$, is a normal distribution with mean

$$
\overline{\delta(r)}=\frac{\gamma \delta}{1-\gamma^{2}}\left(\frac{\psi}{\gamma}+\frac{\nabla^{2} \psi}{u^{2}}\right)-\frac{\bar{x} \sigma_{0}}{1-\gamma^{2}}\left(\gamma \psi+\frac{\nabla^{2} \psi}{u^{2}}\right)
$$

and variance

$$
\begin{aligned}
{[\Delta \delta(r)]^{2}=\sigma_{0}^{2}\left\{1-\frac{1}{\left(1-\gamma^{2}\right)}\left[\psi^{2}+\left(2 \gamma \psi+\frac{\nabla^{2} \psi}{u^{2}}\right) \frac{\nabla^{2} \psi}{u^{2}}\right]\right.} & -5\left(\frac{3 \psi^{\prime}}{u^{2} r}-\frac{\nabla^{2} \psi}{u^{2}}\right)^{2}-\frac{3\left(\psi^{\prime}\right)^{2}}{\gamma u^{2}} \\
& \left.+\frac{(\Delta x)^{2}}{\left(1-\gamma^{2}\right)^{2}}\left(\gamma \psi+\frac{\nabla^{2} \psi}{u^{2}}\right)^{2}\right\} .
\end{aligned}
$$

This enables us to perform the integration over $\tilde{\delta}$ on the right hand-side of equation (A7) also analytically, obtaining

$$
N_{p k}^{n e s t}\left(R \rightarrow R^{\prime}, \delta \rightarrow \delta^{\prime}\right) d R d R^{\prime}=\frac{M\left(R^{\prime}\right)}{\langle\rho\rangle} N\left(R^{\prime}, \delta^{\prime}\right) d R d R^{\prime} \int_{0}^{1} 3 r^{2} N_{p k}\left(R, \delta \mid R^{\prime}, \delta^{\prime}, r\right) d r,
$$

where

$$
N_{p k}\left(R, \delta \mid R^{\prime}, \delta^{\prime}, r\right) d R=\frac{H\left[\tilde{\gamma}(r), \tilde{x}_{*}(r)\right]}{(2 \pi)^{2} R_{*}^{3}} \frac{\exp \left\{-\frac{\left[\nu-\epsilon(r) \nu^{\prime}(r)\right]^{2}}{2\left[1-\epsilon^{2}(r)\right]}\right\}}{\left[1-\epsilon^{2}(r)\right]^{1 / 2}} \frac{\sigma_{2}}{\sigma_{0}} R d R
$$

is the conditional density of peaks with $\delta$ on scales $R$ to $R+d R$ subject to being located at a separation $r$ from a peak with $\delta^{\prime}$ on scales $R^{\prime}$ to $R^{\prime}+d R^{\prime}$. In equation (A11) $R_{*} \equiv \sqrt{3} \sigma_{1} / \sigma_{2}$, and $H\left[\tilde{\gamma}(r), \tilde{x}_{*}(r)\right]$ is defined just as $H\left[\tilde{\gamma}, \tilde{x}_{*}\right]$ in Paper I (eq.[17]), but for the $r$ dependence in $\tilde{\gamma}(r)$ and $\tilde{x}_{*}(r)$ introduced through

$$
\epsilon(r) \equiv \epsilon\left\{1-\frac{\left[\Delta \delta^{\prime}(r)\right]^{2}}{\sigma_{0}^{2}\left(R^{\prime}\right)}\right\}^{1 / 2}, \quad \nu^{\prime}(r)=\frac{\overline{\delta^{\prime}(r)}}{\sigma_{0}\left(R^{\prime}\right)}\left\{1-\frac{\left[\Delta \delta^{\prime}(r)\right]^{2}}{\sigma_{0}^{2}\left(R^{\prime}\right)}\right\}^{-1 / 2} .
$$

Therefore, by defining

$$
N_{p k}^{n e s t}\left(R, \delta \mid R^{\prime}, \delta^{\prime}\right) \equiv \int_{0}^{1} 3 r^{2} N_{p k}\left(R, \delta \mid R^{\prime}, \delta^{\prime}, r\right) d r
$$

equation (A10) adopts the desired compact form similar to equation (3)

$$
N_{p k}^{n e s t}\left(R \rightarrow R^{\prime}, \delta \rightarrow \delta^{\prime}\right) d R d R^{\prime}=\frac{M\left(R^{\prime}\right)}{\langle\rho\rangle} N\left(R^{\prime}, \delta^{\prime}\right) d R^{\prime} N_{p k}^{n e s t}\left(R, \delta \mid R^{\prime}, \delta^{\prime}\right) d R .
$$




\section{REFERENCES}

Bardeen, J.M., Bond, J.R., Kaiser, N., \& Szalay, A.S. 1986, ApJ, 304, 15 (BBKS)

Bernardeau, F. 1994, ApJ, 427, 51

Bond, J.R., Cole, S., Efstathiou, G., Kaiser, N. 1991, ApJ, 379, 440

Bond, J.R., \& Myers, S.T. 1996, ApJS, 103, 41

Bower, R.J., 1991, MNRAS, 248, 332

Lacey, C., \& Cole, S. 1993, MNRAS, 262, 627 (LC93)

Lacey, C., \& Cole, S. 1994, MNRAS, 271, 676 (LC94)

Manrique, A., \& Salvador-Solé, E. 1995, ApJ, 453, 6 (Paper I)

Manrique, A., \& Salvador-Solé, E. 1996, ApJ, 467, 504 (Paper II)

Navarro, J., Frenk, C., \& White, S.D.M. 1996, ApJ, 462, 563

Press, W.H., \& Schechter, P. 1974, ApJ, 187, 425 (PS)

Regös, E., \& Szalay, A.S., 1995, MNRAS, 272, 447

Salvador-Solé, Solanes, J.M., \& Manrique, A. 1997, ApJ, submitted 


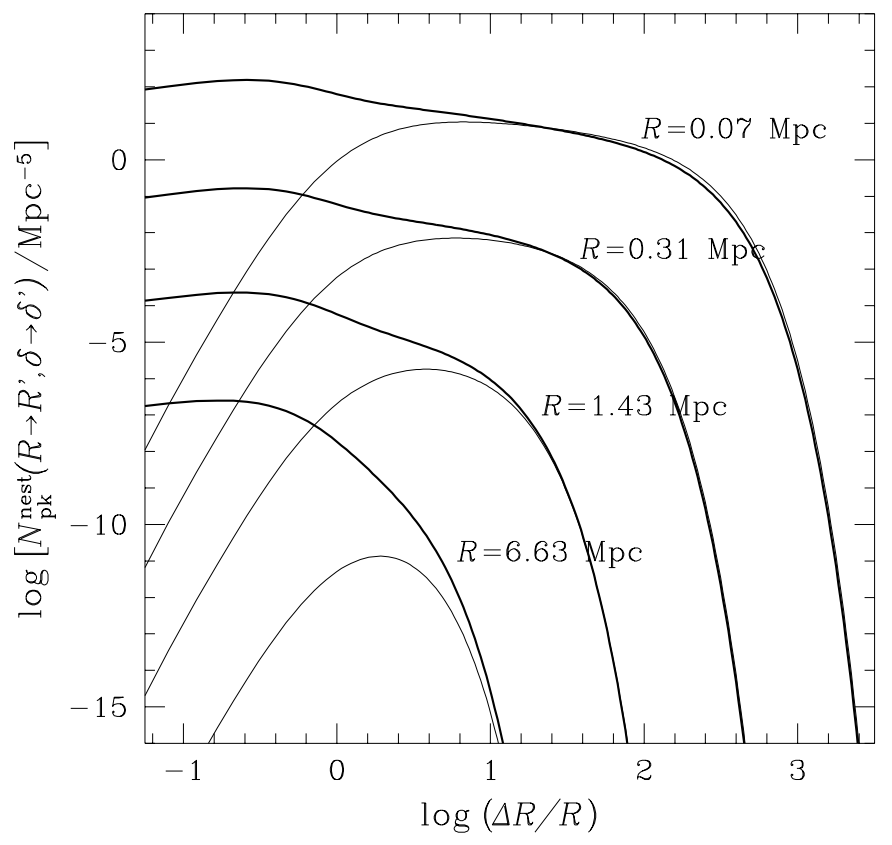

Fig. 1. - Comparison between the old (thin solid lines) and new (thick solid lines) mean density of peaks with $\delta$ on scale $R$ nested within peaks with $\delta^{\prime}=\delta \equiv \delta_{c 0}$ on a larger scale $R^{\prime} \equiv R+\Delta R$. The curves represent different values of $R$ (corresponding to the same masses plotted in Fig. 4). The cosmogony assumed in all Figures to illustrate the behavior of the model is an Einstein-de Sitter universe with a $n=-2$ scale-free power spectrum of density fluctuations normalized to $\sigma_{0}\left(8 h^{-1}\right.$ $\mathrm{Mpc})=.5$. 


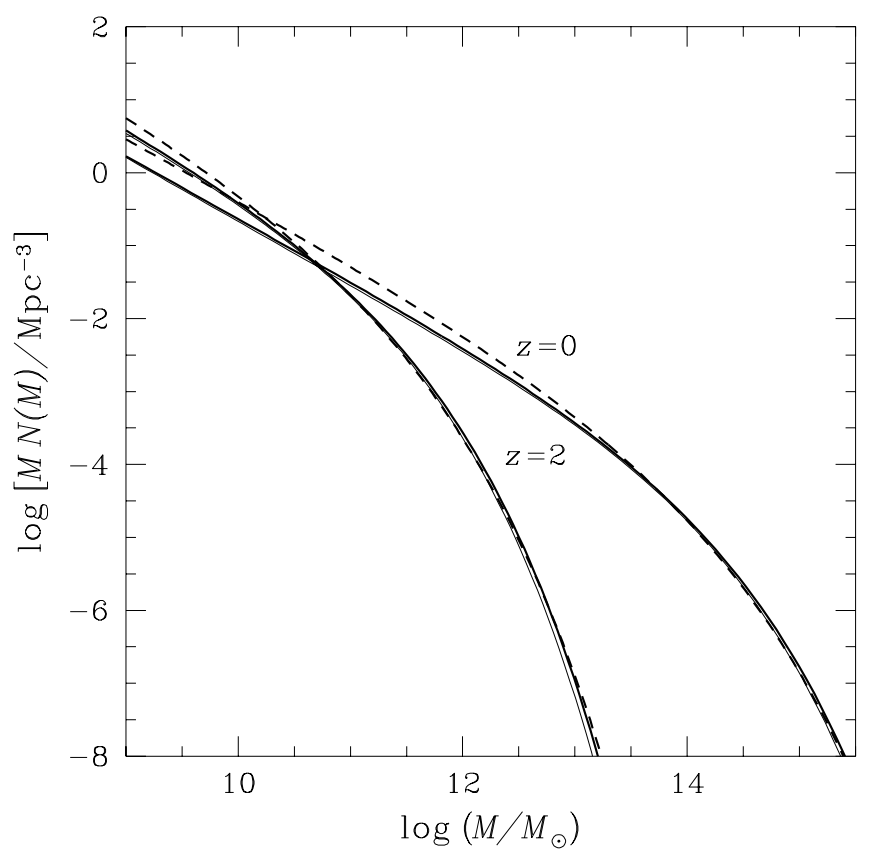

Fig. 2. - Mass functions at two different epochs predicted by the CUSP model using the old (thin solid lines) and new (thick solid lines) approximations for the density of nested peaks and by the LC model (thick dashed lines). The curves corresponding to the old and new versions of the CUSP model are almost superimposed. 


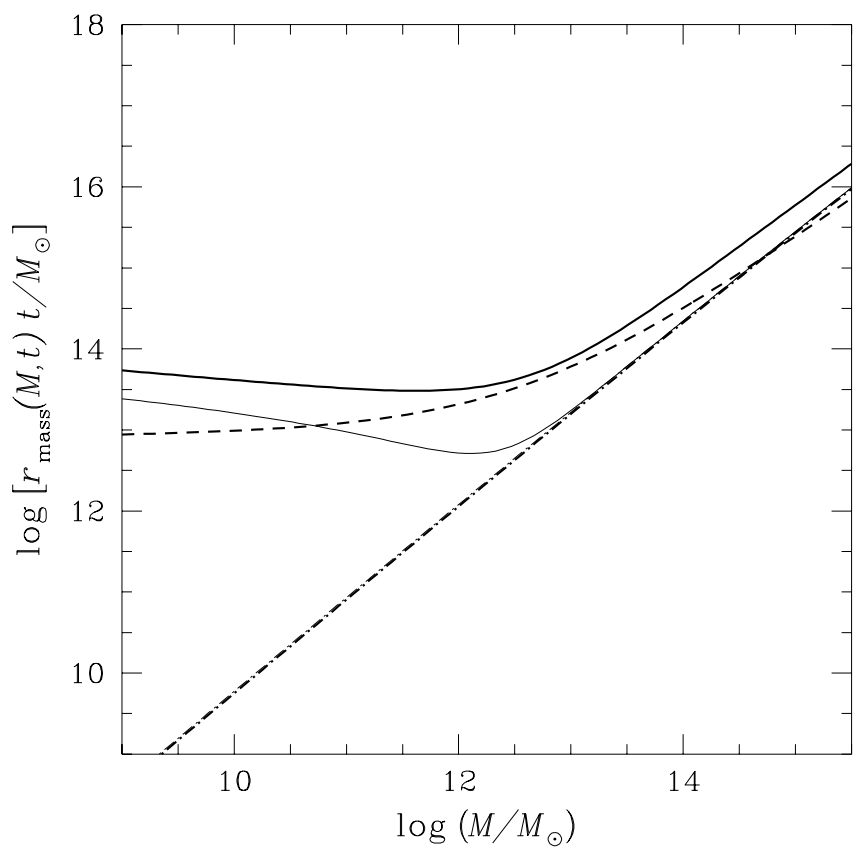

Fig. 3.- Mass accretion rates at $z=0$ predicted by the CUSP model using the old (thin dot-dashed line) and new (thick dot-dashed line) density of nested peaks; both curves almost fully superpose. Also plotted are the total mass increase rates predicted by the old (thin solid line) and new (thick solid line) version of the CUSP model compared with the prediction by the LC model (thick dashed line). 


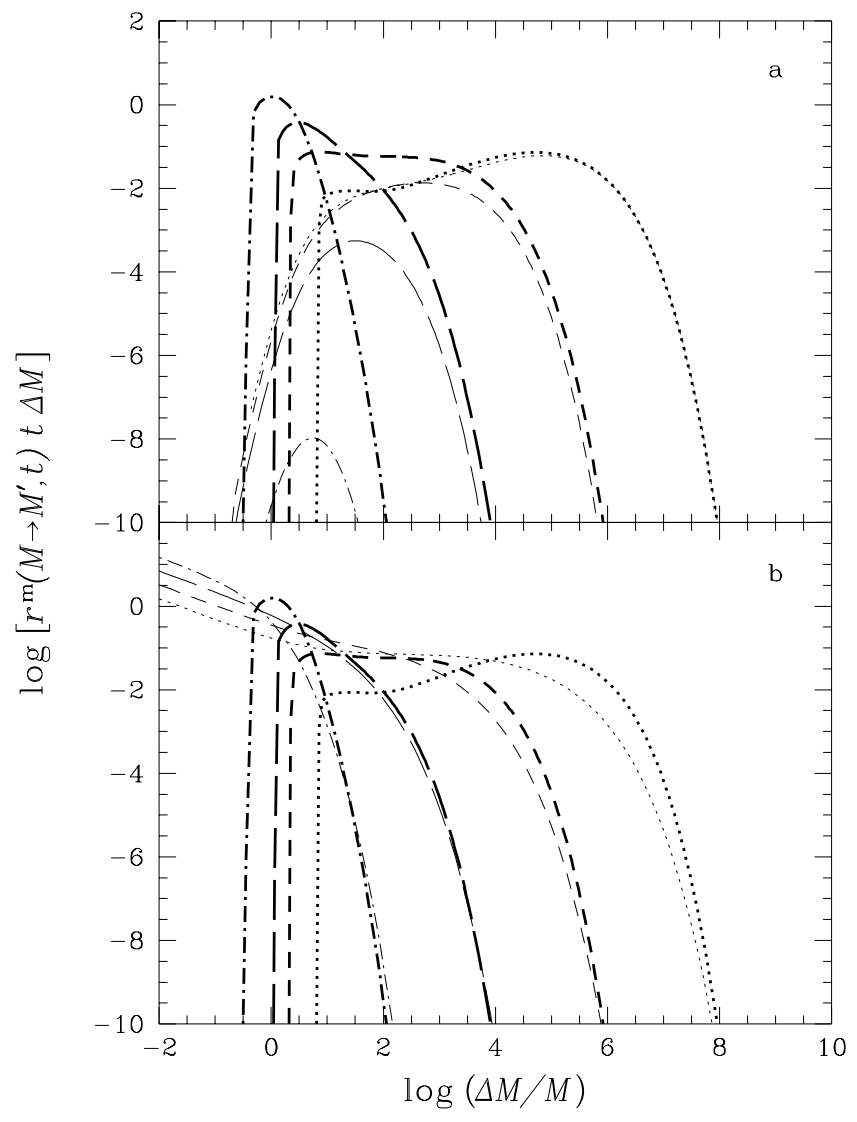

Fig. 4.- Instantaneous specific merger rates at $z=0$ of halos for $M$ equal to $10^{9} \mathrm{M}_{\odot}$ (dotted lines), $10^{11} \mathrm{M}_{\odot}$ (dashed lines), $10^{13} \mathrm{M}_{\odot}$ (long dashed lines), and $10^{15} \mathrm{M}_{\odot}$ (dot-long dashed line) as a function of the relative captured mass $\Delta M / M$. (a) predictions by the CUSP model using the old (thin lines) and new (thick lines) approximations for the density of nested peaks. (b) these latter solutions (thick lines) are compared to the specific merger rates predicted by the LC model (thin lines). 